\title{
MADMAX: Post-Inflationary Axion Dark Matter Search
}

\author{
Chang Lee, on behalf of the MADMAX collaboration \\ Max-Planck-Institut für Physik (Heisenberg-Institut), Föhringer Ring 6 München 80805, Germany
}

\begin{abstract}
Relic axions generated after cosmic inflation may have a mass of around $100 \mu \mathrm{eV}$. While being excellent candidates for cold dark matter, axions in this mass range are seldom covered by existing research based on resonant RF cavities. The dielectric haloscope is a promising alternative technique for the search of dark matter axions in this mass range. The MADMAX collaboration tries to build a dielectric haloscope that is sensitive to post-inflationary QCD axions. The principle, current status, and future plans of the MADMAX experiment are presented.
\end{abstract}

Keywords: dark matter, axion, axion-like particles, WISP, dielectric haloscope

DOI: 10.31526/ACP.NDM-2020.27

\section{INTRODUCTION}

The identity of dark matter remains enigmatic. Among the prominent candidates for dark matter, weakly interacting slim particles (WISPs) are gathering recent attention [1]. These light bosons naturally arise from many extensions of the standard model (SM) that introduce a scalar field. When the symmetry spontaneously breaks, the field forms a "wine-bottle bottom" potential. Oscillations around the trough of the potential correspond to a light Nambu-Goldstone (NG) boson whose interaction with the SM particles is inversely proportional to the symmetry-breaking energy scale. When the scale is big, these WISPs remain elusive. The WISPs could have been generated in the early universe as the primordial plasma cooled down. Once created, they remain stable, as they are very light and barely interact. Because they are not produced via thermal freeze-out, WISPs may be nonrelativistic despite being light. WISPs include axions, axion-like-particles (ALP), and hidden photons. The axion is the most well-known example of WISP. It is originally motivated to solve the strong CP problem. QCD contains a CP-violating term whose strength is parametrized by an angle $\theta$. Experimentally, $\theta$ is known to be smaller than $10^{-10}$ [2]. This extremely small value called for an explanation. Peccei and Quinn proposed to extend the SM with a new global color-anomalous U(1) symmetry that spontaneously breaks at an energy scale $f_{a}$. The QCD anomaly explicitly breaks the symmetry, or "tips" the wine-bottle bottom potential, creating a minimum exactly at $\theta=0$. In this mechanism, $\theta$ becomes a dynamical field, but its expectation value is zero, thus this mechanism solves the strong CP problem [3]. An oscillation around the minimum is the axion, the light pseudo-NG boson of the new field.

A wide range of axion mass $m_{a}$ is allowed. Observation of stellar cooling [4] excludes $f_{a}$ below $3 \times 10^{8} \mathrm{GeV}$. This translates into possible $m_{a}$ below $0.02 \mathrm{eV}$ via axion's coupling with pion [5]. The mass can be further confined if we assume that most of axions were generated via the vacuum realignment mechanism after the cosmic inflation. In this post-inflation scenario, $\theta$ from all causally connected patches of the universe would be averaged. Recent lattice QCD calculation reports that the axion mass in this scenario has to be above $26 \mu \mathrm{eV} \mathrm{[6].} \mathrm{This} \mathrm{range,} \mathrm{however,} \mathrm{is} \mathrm{slightly} \mathrm{higher} \mathrm{than} \mathrm{the} \mathrm{optimal} \mathrm{range} \mathrm{of} \mathrm{most} \mathrm{current} \mathrm{axion} \mathrm{search} \mathrm{experiments}$ that use resonant cavities $[7,8]$. A dielectric haloscope $[9,10,11,12]$ is designed to optimally detect axion and WISP dark matter around $100 \mu \mathrm{eV}$.

\section{DIELECTRIC HALOSCOPE}

A dielectric haloscope relies on the interaction of axions with two photons. The Maxwell equations are modified with the axion field and provide an interaction term [13]:

$$
\mathcal{L}=-\frac{\alpha}{2 \pi} C_{a \gamma} \mathbf{E} \cdot \mathbf{B} \theta
$$

Here, $\alpha$ is the fine structure constant, and $C_{a \gamma}$ is a model-dependent coupling constant between the axion and photons. If one of the photons is replaced by a static magnetic field, the interaction appears as an axion being converted into a photon. A $100 \mu \mathrm{eV}$ axion will become a microwave photon of $24 \mathrm{GHz}$. Because we assume a cold dark matter axion, kinetic energy is subdominant to the rest mass, and the resulting microwave will appear as a mono-energetic power peaked above noise background. The electric field of the axion-induced photon is

$$
\mathbf{E}_{0}=-\frac{\alpha}{2 \pi \epsilon} C_{a \gamma} B_{e} \theta(t),
$$

where $B_{e}$ is the external magnetic field, and $\epsilon$ is the relative permittivity of the material. Unfortunately, this signal power is extremely small, as signal power from a flat metallic mirror immersed in a 10-T field is only $2.2 \times 10^{-27} \mathrm{~W} / \mathrm{m}^{2}$ [13], far below the sensitivity of modern technology.

The signal power can be boosted using layers of dielectrics. At the boundary between different media, the change of $\epsilon$ leads to discontinuity of the axion-induced electric field $E_{0}$. To amend this discontinuity, two traveling waves are emitted away from the 

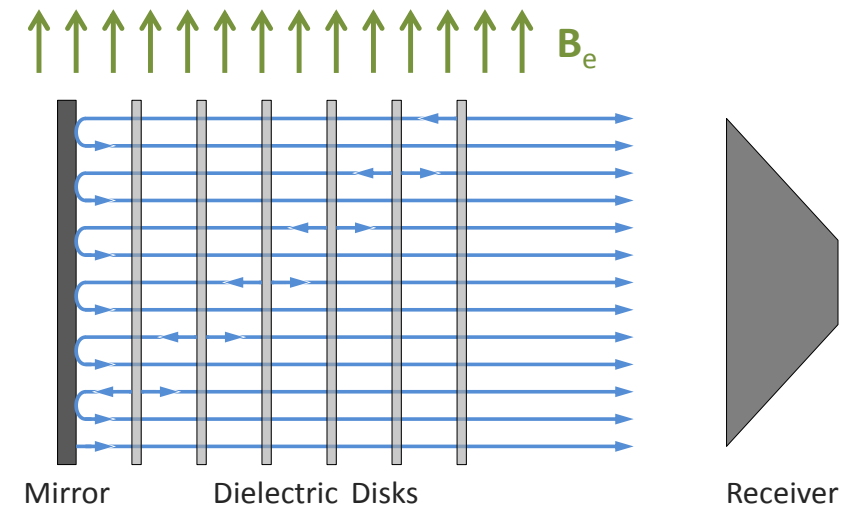

Receiver

FIGURE 1: Schematic of a dielectric haloscope. Image from [10]

boundary. These are the axion-induced photons described above. The signal power can be boosted by adding more layers of dielectric as shown in Fig. 1. Constructive interference among the signals can further boost the signal power at the frequency of interest. The ratio of the boosted electric field to $E_{0}$ is parametrized by the boost factor $\beta$. The boosted signal is received by a microwave receiver chain, which consists of an antenna and an amplifier. To scan a wide axion mass range, the boost frequency can be tuned by controlling the gaps between the dielectrics. Contrary to the resonant cavities, a single large volume can be instrumented even at high frequency. The signal power can be boosted by more than a factor of 20,000 compared with the single mirror, enabling us to probe the post-inflationary QCD axion. The scan rate [10] of the dielectric haloscope is

$$
\frac{\Delta t}{1.3 \text { days }} \sim\left(\frac{\mathrm{SNR}}{5}\right)^{2}\left(\frac{400}{\beta}\right)^{4}\left(\frac{1 \mathrm{~m}^{2}}{A}\right)^{2}\left(\frac{m_{a}}{100 \mu \mathrm{eV}}\right)^{2}\left(\frac{T_{\text {sys }}}{8 \mathrm{~K}}\right)^{2}\left(\frac{10 \mathrm{~T}}{B_{e}}\right)^{4}\left(\frac{0.8}{\eta}\right)^{2} C_{\alpha \gamma}^{-4} .
$$

Here, SNR is the signal-to-noise ratio, $\eta$ is the signal collection efficiency, and $T_{s y s}$ is the system noise temperature.

\section{CURRENT STATUS}

Research and development are ongoing to realize the principle of the dielectric haloscope. The shape and power of the axioninduced beam is studied using a full 3D simulation [14]. The simulation informs how the boost factor might vary depending on the disk spacing, mechanical imperfection, and coupling to the antenna. A room-temperature proof-of-principle setup is built to compare the measurement with simulation. The setup consists of a copper mirror, up to $2020 \mathrm{~cm}$-diameter sapphire disks, a focusing reflector, and a horn antenna. Group delay measured from up to five disks agrees with the simulation [15]. Recent updates of the antenna and disk holders allow more precise control of the system, so that more disks can be inserted. The system noise temperature of a dielectric haloscope will be complicated as it reflects most on incident power. This includes noise from the amplifier, which in turn interferes with itself. To study the variation of the noise temperature, a small dielectric haloscope is built. Its disks are spaced to be strongly resonant so that the thermal noise from the haloscope is close to the boost factor. Measurement of the thermal signal would help us understand the boost factor of the dielectric haloscope. Last, quantum-noise-limited amplifiers are being developed to lower the amplifier noise temperature. A traveling wave parametric amplifier (TWPA) developed by Institut NÉEL showed a noise temperature around $1 \mathrm{~K}$ at $6 \mathrm{GHz}$ [16], close to the quantum noise limit. A modified version that works at 10-15 GHz will be delivered this year.

A prototype will serve as a technical test platform for the final MADMAX. The prototype haloscope consists of 20 sapphire disks of $30 \mathrm{~cm}$ diameter. Disk-positioning mechanisms based on piezo motors and laser interferometers at cryogenic temperature will be tested. Once the cryogenic tests are finished, the prototype will be moved to CERN, where it will be fitted inside the Morpurgo magnet [17] in 2024. The preliminary axion search data will be taken using the magnet's $1.6 \mathrm{~T}$ field. The cyan layers in Fig. 2 show the projected sensitivity. Here, the axion/ALP-photon coupling strength is drawn as a function of the axion/ALP mass $m_{a}$. For comparison with the conventional results, $g_{a \gamma}=\frac{\alpha}{2 \pi f_{a}} C_{a \gamma}$ is used. For the $70-90 \mu \mathrm{eV}$ search range, the limit assumes $50 \mathrm{MHz}$ measurement bandwidth, one day tuning time, $8 \mathrm{~K}$ system noise temperature, $50 \%$ efficiency, $5 \sigma$ signal power above noise level, and 90 days measurement time.

The final MADMAX will be sensitive to the QCD axion range. This requires a dielectric haloscope that consists of $80 \mathrm{LaAlO}_{3}$ (relative permittivity $\varepsilon \approx 24$, loss $\tan \delta \approx 10^{-5}$ ) disks of $1.2 \mathrm{~m}$ diameter. A superconducting dipole magnet will supply a $9 \mathrm{~T}$ field in its $1.35 \mathrm{~m}$ warm bore. The magnet is currently being designed by European Innovation partners CEA Saclay and Bilfinger Noell. The design will be finished by 2023, and the final magnet will be delivered by 2025 to the H1 North hall of DESY, where it will replace the H1 magnet and reuse the existing yoke. The final MADMAX will start from 2026, and its projected sensitivity for axionphoton coupling is shown as the till layers in Fig. 2. For the 50-200 $\mu \mathrm{eV}$ search range, the upper "conservative" limit assumes $50 \mathrm{MHz}$ measurement bandwidth, one day tuning time, $8 \mathrm{~K}$ system noise temperature, and three years of measurement time. The lower "optimistic" limit assumes $20 \mathrm{MHz}$ measurement bandwidth, one day tuning time, $4 \mathrm{~K}$ system noise temperature, and five years of measurement time. This measurement can carve into the yellow band that is favored by various QCD axion models, including the KSVZ model (red line) [19]. DFZS [20], another benchmark model, is marked by the blue line. Also shown are limits 


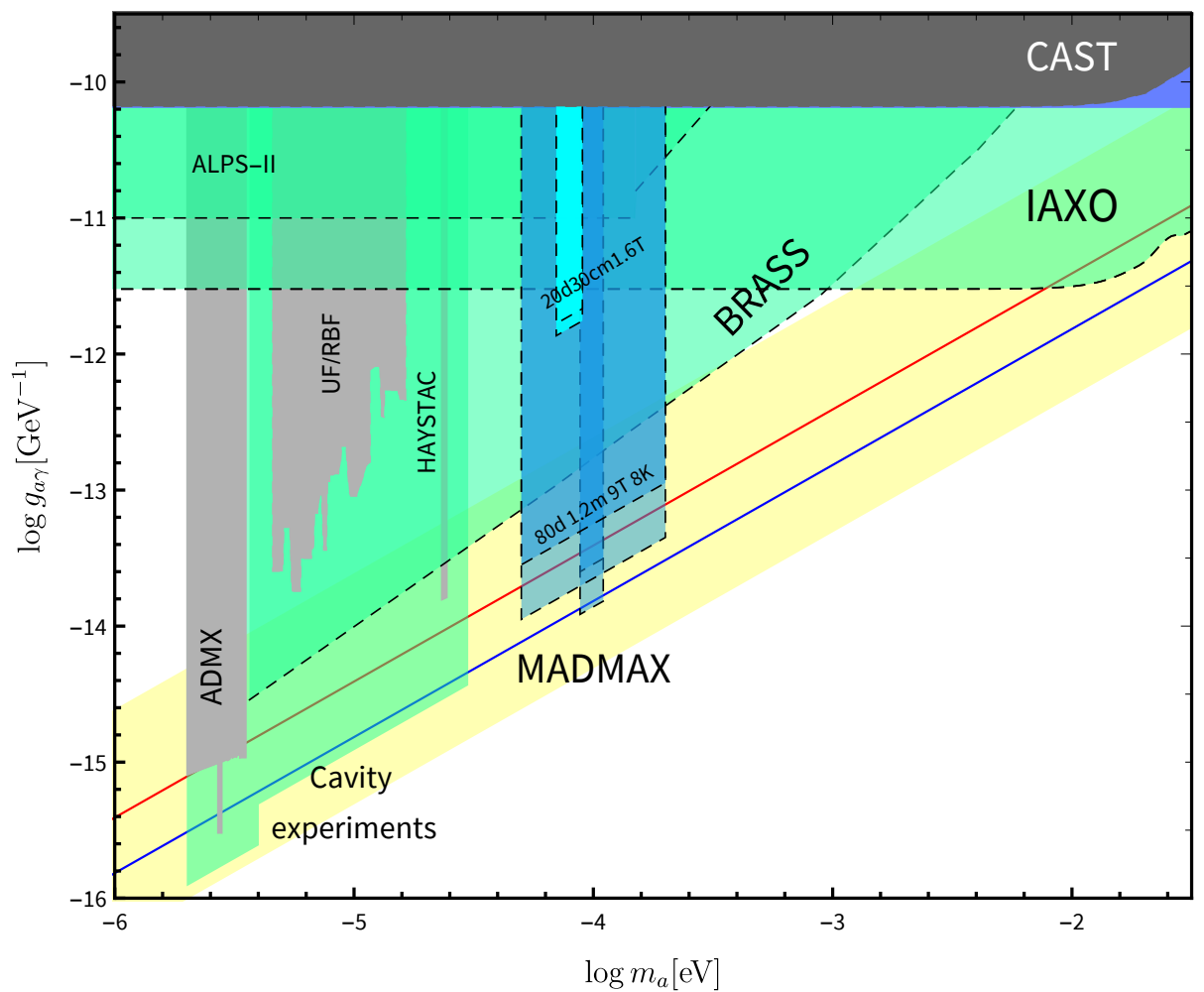

FIGURE 2: Projected and measured axion-photon coupling $\left(g_{\alpha \gamma}\right)$ sensitivities of the MADAMX and other experiments as a function of axion/ALP mass $m_{a}$. See the text for details. Image from [18]

and projections from helioscopes [21, 22], light-shining-through-wall experiments [23], resonant cavities [7, 8], and dish antenna experiments [13].

\section{References}

[1] J. Jaeckel and A. Ringwald, Annu. Rev. Nucl. Part. Sci. 60, 405-437 (2010), arXiv:1002.0329.

[2] C. Abel et al., Phys. Rev. Lett. 124, 081803 (2020), arXiv:2001.11966.

[3] R. D. Peccei and H. R. Quinn, Phys. Rev. Lett. 38, 1440--1443 (1977).

[4] G. Raffelt, Lect. Notes Phys. 74151 (2008).

[5] G. Grilli di Cortona et al., J. High Energy Phys. 1601034 (2016), arXiv:1511.02867.

[6] S. Borsanyi et al., Nature 539, no. 7627, 69 (2016) doi:10.1038/nature20115, arXiv:1606.07494.

[7] N. Du et al., Phys. Rev. Lett. 120, 151301 (2018), arXiv:1804.05750.

[8] B. Brubaker et a l., Phys. Rev. Lett. 118, 061302 (2017), arXiv:1801.00835.

[9] D. E. Morris, Report No. LBL-17915, (1984).

[10] A. Caldwell et al., Phys. Rev. Lett. 118, 091801 (2017), arXiv:1611.05865.

[11] A. J. Millar et al., J. Cosmol. Astropart. Phys. 2017, 01 (2017), arXiv:1612.07057.

[12] MADMAX Collaboration, Eur. Phys. J. C79, 3 (2019), arXiv:1901.07401.

[13] D. Horns et al., J. Cosmol. Astropart. Phys. 2013016 (2013), doi:10.1088/1475-7516/2013/04/016, arXiv:1212.2970, http://www.iexp.uni-hamburg.de/groups/astroparticle/brass/brassweb.html

[14] S. Knirck et al., J. Cosmol. Astropart. Phys. 2019, 026 (2019), arXiv:1906.02677, J. Schütte-Engel, The 14th Patras Workshop on Axions, WIMPs and WISPs, DESY (2018), arXiv:1811.00493.

[15] J. Egge et al., Eur. Phys. J. C80, 1-10 (2020), arXiv:2001.04363.

[16] L. Planat et al., Phys. Rev. X10, 021021 (2020), arXiv:1907.10158.

[17] M. Morpurgo, Cryogenics 19, 411 (1979).

[18] MADMAX Collaboration, arXiv:2003.10894.

[19] J. E. Kim, Phys. Rev. Lett. 43103 (1979), M. A. Shifman, A. I. Vainshtein and V. I. Zakharov, Nucl. Phys. B 166493 (1980).

[20] A.P. Zhitnitskii, Sov. J. Nucl. Phys. 31260 (1980),

M. Dine, W. Fischler and M. Srednicki, Phys. Lett. B 104199 (1981).

[21] CAST Collaboration, Nature Physics 13, 584 (2017), arXiv:1705.02290.

[22] IAXO collaboration, arXiv:1904.09155.

[23] R. Bähre et al., J. Inst. 8, T09001 (2013), arXiv:1302.5647. 